\title{
Об использовании метода реальных опционов в электроэнергетике
}

\author{
Дранев Ю.Я. ${ }^{31}$
}

Привлечение инвестищий является одной из ключевых задач, решение которых необходимо для продолжения развития сектора российской электроэнергетики. Высокая неопределенность, связанная с ограничениями на рост тарифов и волатильностью иен на энергоносители, отрицательно влияет на инвестиционный климат в отрасли. Однако метод реальных опџионов позволяет учесть управленческую гибкость в резко меняюшихся условиях и по-новому взглянуть на риски инвестищий в электроэнергетику.

В данной статье приводится обзор литературы по способам применения реальных опщионов для оченки инвестиционных проектов и принятия управленческих решений. $B$ частности, рассмотрен подход к определению стоимости генерируюших компаний как реальных опщиионов (spark spread options) на электричество со страйками, зависящими от стоимости энергоносителей. Большое внимание исследователей привлекает также учет возможности переключения на другие виды топлива, включая возобновляемые источники энергии. Важную роль в развитии электроэнергетики играет энергоэффективность. Поэтому в обзоре приведены результать работ по оценке инвестиций 8 энергоэффективность с помощьюю метода реальных опџионов.

JEL: G30, H54

Ключевые слова: реальные опџฺионы, спарк спрэд опџฺион, необратимые инвестиции, энергоэффективность, электроэнергетика

\section{Введение}

В конце 2010 года фактически завершился основной этап реформы российской электроэнергетики. Реформа была направлена в первую очередь на привлечение инвестиций в отрасль. Согласно Энергетической стратегии России, выработка электроэнергии до 2030 года должна вырасти в 2 раза. При растущей социальной нагрузке на бюджет государство не может финансировать отрасль в одиночку. Для реализации этих планов необходимо привлечение частного капитала, в том числе зарубежных инвестиций. В период 2006-2008 годов в электроэнергетику России инвестировали сразу несколько крупных компаний, таких как E.ON и Enel. Финансовый кризис 2008 года помешал продолжению этого процесса. В результате за последние два года основные средства на развитие и модернизацию электроэнергетики поступали в основном из бюджета.

В сегодняшних условиях, когда последствия кризиса до конца не устранены, инвесторы в ТЭК сталкиваются в России не только с неопределенностью при резких колебаниях цен на энергоносители, но и с частыми изменениями в регулировании отрасли, связанными с попытками ограничить рост тарифов на электроэнергию. Ключевые этапы реформы, а именно переход на долгосрочный рынок мощности для генерирующих компаний и введение RAB-регулирования тарифов на базе доходности на капитал для распределительных компаний, поначалу были положительно оценены инвесторами. Однако затем снижение темпов роста уровня жизни населения и высокий уровень инфляции привели к тому, что государство ввело дополнительные ограничения на ежегодный рост тарифов и понизило минимальную доходность на капитал в рамках RAB-регулирования. Такие действия приводят к повышению неопределенности и рисков инвестиций в электроэнергетику. Однако

\footnotetext{
${ }^{31}$ Старший преподаватель кафедры экономики и финансов фирмы, НИУ ВШЭ.
} 
снижения инфляционного давления на экономику можно также достичь и путем модернизации электроэнергетики и повышения энергоэффективности, о чем, в частности, говорил Президент РФ в декабре 2010 года. И для этих целей необходимы дополнительные инвестиции.

Многие инвесторы интересуются сектором электроэнергетики, замечая его значительный потенциал роста, в том числе и в России. Так, по стоимости установленной мощности сектор остается сильно недооцененным даже по сравнению с развивающимися странами. Но подсчитывая стоимость компаний сектора, проектов по строительству новых мощностей, сетей, а также модернизации старых объектов, потенциальные инвесторы, использующие традиционный метод дисконтированных денежных потоков (DCF), повышают требуемую доходность на капитал, чтобы учесть все риски подобных проектов. При таком способе оценки проекты зачастую становятся нерентабельными и отвергаются инвесторами. Однако оценка на основе DCF не учитывает управленческой гибкости в изменяющихся условиях. Например, при повышении энергоэффективности и ослаблении инфляционного давления государство может пойти на уступки инвесторам и снять ограничения на рост тарифов. Таким образом, дополнительные инвестиции в энергоэффективность могут повысить привлекательность проектов в электроэнергетике в целом.

В связи с возрастающими потребностями в электроэнергии реформы электроэнергетики, а именно либерализация рынка и приватизация энергетических активов, происходили не только в России, но и во многих других странах, включая Великобританию, США, Францию. Причем происходили эти события относительно недавно. В США принятие в 1992 году Акта об энергетической политике (Energy Policy Act) привело к дерегулированию рынка к 1997 году. Переход к конкурентному рынку сказался и на инвестициях, в том числе на структуре капитала американских электроэнергетических компаний, так что уровень финансового рычага снизился в период реформы на 17\% (Sanyal, Bulan, 2008). По-существу, компании откладывали привлечение долгового финансирования в связи с ростом неопределенности во время переходного периода.

Конечно, не только действия регулятора могут оказать влияние на инвестиции в электроэнергетику. Одним из ключевых параметров для оценки генерирующих компаний является стоимость энергоносителей. При росте цен на энергоносители производство электроэнергии может стать нерентабельным и менеджмент может принять решения о приостановке генерации электричества или отложить предполагаемые инвестиции. Возможность переключения с одного энергоносителя на другой, например с мазута на газ, при колебаниях цен или ужесточении экологических требований позволит повысить привлекательность проекта. Возникает и множество других ситуаций, таких как отсрочка IPO, возможность синергии и т.Д., когда управленческие решения могут повысить стоимость компании или проекта. Для электроэнергетики особенно важно учесть положительный фактор управленческой гибкости, который повысит привлекательность сектора для инвесторов. Отразить этот фактор в оценке позволяет метод реальных опционов.

Понятие реальных опционов было введено Майерсом в 1977 году. С тех пор эта тематика привлекла внимания значительного числа авторов (Dixit, Pindyck, 1994; Trigeorgis, 1996). В конце 1990-х и в 2000-е годы ряд компаний стали использовать метод реальных опционов для оценки. По различным оценкам, от 10 до $27 \%$ крупнейших компаний используют метод реальных опционов при оценке капиталовложений. В одном из недавних исследований (Driouchi, Bennett, 2010) показано, что транснациональные корпорации, использующие методику реальных опционов в своих международных проектах, являются более конкурентоспособными. Так, компании, внедрившие платформы по управлению проектами с учетом управленческой гибкости и применяющие при оценке реальные опционы, снижают возможные риски таких проектов. Причем инвестиции во внедрение практики использования реальных опционов положительно сказываются на операционной деятельности компании по сравнению с конкурентами. 
Такие исследования позволяют предполагать, что внедрение практики использования реальных опционов в российских компаниях, в том числе в секторе электроэнергетики, может привести к снижению потенциальных рисков и оказать положительное влияние на инвестиционный климат в отрасли. В данной обзорной статье будут рассмотрены возможности применения метода реальных опционов для оценки проектов и компаний электроэнергетики, а также принятия инвестиционных решений.

\section{Оценка генерирующих активов}

В связи с острой нехваткой электроэнергии в ряде регионов России привлечение инвестиций в генерацию является одной из приоритетных задач, направленных на развитие инфраструктуры. При оценке генерирующей компании необходимо учитывать два ключевых параметра. Во-первых, это стоимость энергоресурсов, необходимых для генерации электроэнергии, а во-вторых - стоимость самой электроэнергии, от продажи которой складывается выручка. После либерализации рынка электроэнергии во многих странах можно предположить, что цена на электричество изменяется случайно, в соответствии с изменением спроса и предложения.

Использование DCF-метода в условиях значительных изменений цен на энергоносители и электричество не учитывает возможной реакции менеджмента на эти изменения и приводит к искажению оценки генерирующих активов. Применение реальных опционов позволяет учесть возможности менеджмента.

Широко признано в литературе (см. например,:Deng, 2003; Tseng, 2002), что генерирующие активы можно оценивать с помощью реальных опционов, так называемых spark spread options. Идея заключается в том, что генерирующий актив преобразует определенный вид топлива в электричество и «включается», только когда стоимость производимого электричества превышает расходы на топливо и производство энергии. Таким образом, генерирующий актив можно описать как серию колл-опционов на электричество со страйками, зависящими от цены энергоносителя. Так как время исполнения таких опционов является случайным, авторы для построения соответствующих моделей зачастую прибегают к методам динамического программирования.

Нужно отметить, что для моделирования изменения цен на энергоносители и электричество используют либо геометрическое броуновское движение, либо так называемые процессы Орнштейна - Уленбека, возвращающиеся к среднему значению (meanreverting process). Причем некоторые авторы (Benth, 2007), указывают на более сложную структуру изменения цен на электричество. Предложенные ими процессы для моделирования цен позволяют учесть сезонность и скачки, которые наблюдаются на рынке электроэнергии.

Опционы на переключение с одного вида топлива на другой применялись для оценки теплогенерирующих активов (Kulatilaka, 1993). Позднее модели, использующие опционы на переключение, стали применять и для оценки генерирующих активов. Так, в статье Фусс (Fuss, 2010) применен метод реальных опционов для анализа инвестиций в генерирующие мощности, которые могут использовать различные источники энергии, такие как уголь, газ, биомасса и ветер. Авторы упоминают многочисленные риски - рыночные, технологические, социально-экономические и т.п., с которыми сталкивается инвестор. Для оценки оптимального портфеля инвестиций с учетом различных источников энергии Фусс решает задачу минимизации этих рисков. При этом рассматриваются различные сценарии, соответствующие ограничениям по выбросам $\mathrm{CO}_{2}$. Авторы ставят под сомнение нормальность распределения цен на электричество и поэтому для оценки рисков вместо традиционной волатильности используют CVaR (conditional value at risk). Результаты статьи могут быть использованы для принятия решения об инвестициях в электроэнергетику с использованием того или иного энергоресурса.

Проблема оценки генерирующих активов в России связана с дополнительными 
сложностями. Так, помимо продажи электроэнергии на открытом рынке генераторы принимают участие в конкурентном отборе мощности (КОМ), в рамках которого продается мощность по долгосрочным контрактам. Объем мощности и ее стоимость в этом случае определяются рынком и являются дополнительным источником неопределенности. Более того, генерирующие компании берут на себя обязательства по строительству новых мощностей и модернизации старых. Нарушение этих обязательств ведет за собой штрафы и ограничения на продажу электроэнергии и мощности на свободном рынке. Государство также стремится ограничить рост тарифов на электроэнергию, что приводит к регулируемым тарифам в ряде регионов, так как рыночные цены превышают пороговые значения. Это приводит к тому, что часть мощности генерирующих компаний распределяется без конкурса. Все эти факторы неопределенности плохо учитываются при принятии решений на основе оценки проектов по методу DCF. Метод реальных опционов позволяет смоделировать возможные действия менеджмента как в случае дополнительных ограничений на рост тарифов, так и при изменениях цен на энергоносители и электроэнергию.

\section{Поиск оптимального времени инвестирования}

Инвестиции в проекты по строительству новых объектов электроэнергетики и ремонт старых зачастую являются необратимыми (irreversable investment). Даже если в будущем условия стали неблагоприятными для реализации такого проекта, выход из него потребует больше затрат, чем его завершение. В некоторых случаях обязательства, взятые на себя инвесторами, должны быть выполнены даже в случае ухудшения конъюнктуры рынка. В таких условиях инвестор, с одной стороны, стремится максимально откладывать сроки реализации проекта, чтобы снизить неопределенность и потенциальные риски проекта. С другой стороны, конкуренция или обязательства перед государством не позволяют значительно переносить сроки проекта. Тематике необратимых инвестиций посвящено большое количество исследований (Pindick, 1988). Возникает задача поиска оптимального времени инвестирования, которая может быть решена с помощью метода реальных опционов.

Остановимся на применении метода реальных опционов для оценки необратимых инвестиций на примере Японии. Японская электроэнергетика на 70\% состоит из атомной энергетики. Ядерные реакторы начинают устаревать. Процесс модернизации атомной электростанции путем замены реактора является чрезвычайно капиталоемким и необратимым. Наито и соавторы (Naito et all., 2009) предполагают, что решение о замене реактора принимается при определенном уровне цен на электроэнергию, динамика которых определяется геометрическим броуновским движением. Замена реактора представляется в виде двух реальных опционов: на ликвидацию старого оборудования и строительство и установку нового, причем определенная в статье авторами цена опциона на ликвидацию оказывается ниже, чем цена вложенных опционов на ликвидацию и строительство нового реактора.

Как уже отмечалось, в России зачастую инвесторы связаны с государством определенными обязательствами. Государство, чтобы привлечь дополнительные инвестиции в сектор электроэнергетики, либо софинансирует часть проектов, либо стимулирует инвестиции, обеспечивая определенную доходность на капитал, как в рамках RABрегулирования для сетевых компаний, так и в рамках долгосрочного рынка мощности для генерирующих компаний. Однако по условиям программ стимулирования строительства новых и модернизации старых объектов электроэнергетики компании обязуются соблюдать объемы инвестиций и сроки выполнения работ. В случае резкого роста ставок на финансовом рынке, например в условиях финансового кризиса в России, требуемая инвесторами доходность на капитал также увеличивается. При этом минимальные уровни доходности на капитал, установленные государством, становятся неинтересны инвесторам. Но возможные штрафы и опасность потери доли рынка заставляют электроэнергетические 
компании продолжать финансировать проекты. Таким образом, уровень процентных ставок является еще одним источником неопределенности для необратимых инвестиций в секторе электроэнергетики.

\section{Использование возобновляемых источников энергии}

Возобновляемые источники энергии, такие как ветряная энергия, солнечная энергия и малая гидроэнергетика, начинают все чаще использоваться в электроэнергетике. По прогнозам Международного энергетического агентства, их доля при генерации электроэнергии к 2030 году может вырасти до 30\%. Основной причиной роста популярности возобновляемых источников энергии являются ограничения на выброс вредных веществ в рамках Киотского протокола и других межправительственных соглашений. Генерирующие мощности, работающие на угле или мазуте, выбрасывают значительное количество $\mathrm{CO}_{2}$ в атмосферу. Так как в большинстве развитых стран существуют квоты на выброс углекислого газа, то таким предприятиям приходится платить за выбросы. Причем стоимость выбросов определяется на свободном рынке. Таким образом, в деятельности генерирующих компаний, работающих на угле, мазуте и даже газе, возникает еще один источник неопределенности, связанный с оплатой квот на выбросы углекислого газа.

Многие авторы (Verbruggen, 2009; Menegaki, 2007) и другие касаются в своих исследованиях использования возобновляемых источников энергии в связи с ограничениями по выбросам парниковых газов в развитых странах. Компании, которые инвестируют в проекты в электроэнергетике, связанные с использованием возобновляемых источников, сталкиваются не только с рисками изменения цен на электричество и энергоносители, но и получают дополнительную выгоду от государственного стимулирования использования возобновляемых источников энергии. Авторы особенно подчеркивают возникающую в этом случае стоимость управленческой гибкости в условиях неопределенности, которую позволяет учесть метод реальных опционов.

Часть исследований посвящено использованию биотоплива в электрогенерации. При этом метод реальных опционов также является ключевым для принятия решений. Так, Шмит (Schmit, 2009) использует реальные опционы по выходу из проекта для анализа инвестиций в завод по производству электроэнергии из биомассы.

Для России в ближайшие несколько лет проблема загрязнения атмосферы топливноэнергетическим комплексом не является настолько острой. Использование возобновляемых источников энергии, за исключением гидроэлектростанций, пока значительно уступает в рентабельности станциям, работающим на газе и даже мазуте. Компания «Русьгидро» занимается инвестициями в альтернативную энергетику, в том числе ветроэнергетику, но пока объем этих инвестиций незначительный и они носят скорее экспериментальный характер. Вопрос о необходимости наращивания использования возобновляемых источников энергии в России остается открытым. Так, по прогнозам ИНП РАН, уже к 2015 году Россия может превысить квоты на выбросы $\mathrm{CO}_{2}$ в рамках Киотского протокола. В этом случае у российских компаний возникнут проблемы, схожие с проблемами компаний в развивающихся странах, и появятся стимулы к переходу на возобновляемые источники энергии.

\section{Выбор поставщика электроэнергии}

После либерализации рынка электроэнергии в России, как и во многих других странах, встает вопрос о поиске оптимального набора поставщиков электроэнергии. Для выбора поставщика электроэнергии необходимо не только учитывать, по какой цене будет заключен контракт, но и бесперебойность снабжения электричеством, так как многие объекты инфраструктуры, такие как аэропорты, например, не должны испытывать сложности с неожиданным отключением электроэнергии. 
Хики (Hickey, 2010) отмечает недостатки портфельной теории при оценке стоимости электричества из различных источников для конечного потребителя. Авторы упоминают альтернативный вариант поиска «оптимального портфеля» контрактов на электричество с помощью теории реальных опционов, которая учитывает управленческую гибкость в условиях сильного колебания цен на энергоносители и возможные перебои в поставках.

\section{Повышение энергоэффективности}

Помимо бесперебойного электроснабжения одной из ключевых проблем российский потребителей является низкая энергоэффективность и слабое внедрение энергосберегающих технологий. В развитых странах проблемы энергоэффективности тесно связаны с вопросами вредных выбросов в атмосферу. Ограничения по выбросам стимулируют компании повышать энергоэффективность и инвестировать в энергосберегающие технологии. Для оценки целесообразности таких инвестиций требуется учесть множество факторов неопределенности. Ли и Хуан (Lin, Huang, 2009) существенно усложнили модели по оценке инвестиций в энергосбережение на предприятии. Так, авторы включили в модель не только реальный опцион на отсрочку инвестирования в энергосбережение, но и учли возможность обновления оборудования, а также приостановки его использования. Для моделирования случайного характера изменения цен авторы используют процесс геометрического броуновского движения с пуассоновскими скачками. Таким образом, в статье предлагается учитывать не только колебания цен на электроэнергию с определенной волатильностью, но и резкие скачки цены в случае экстраординарных событий.

Высокие цены на углеводородные энергоносители являются одной из ключевых проблем электроэнергетики во всем мире. Однако, по прогнозам МЭА, до 2030 года потребление углеводородов развитыми странами практически не изменится в условиях роста экономики этих стран. Причем лишь отчасти этот процесс связан с переходом на возобновляемые источники энергии. Как отмечает МЭА, повышение энергоэффективности и энергосберегающие технологии, такие как комбинирование генерации тепла и электроэнергии, значительно снизили зависимость развитых стран от резких скачков цен на углеводородное топливо.

В России в ближайшие годы планируется либерализовать цены на газ. В связи с авариями на угольных шахтах происходят скачки цен на уголь. Цены на мазут на внутреннем рынке сильно зависят от изменения мировых цен на нефть. Все эти процессы оказывают негативное влияние на инвестиционную привлекательность российской электроэнергетики, особенно в условиях жестких ограничений на рост тарифов. Инвестиции в энергосберегаюшие технологии и повышение энергоэффективности могли бы снизить зависимость потребителей от цен на электроэнергию и позволить государству снизить регулирование сектора. Оценка таких инвестиций методом реальных опционов учитывает множество факторов неопределенности.

\section{Заключение}

Перспективы роста спроса на электроэнергию в России в связи с развитием экономики указывают на необходимость улучшения инвестиционного климата в отрасли. Включение в оценку инвестиций управленческой гибкости в условиях высокой неопределенности является важнейшим фактором для привлечения инвестиций в капиталоемкий сектор электроэнергетики. В литературе признаны преимущества метода реальных опционов, учитывающих управленческую гибкость, перед методом DCF. Оценка генерирующих компаний как реальных опционов на электроэнергию с возможностью остановки, принятие решений об отсрочке необратимых инвестиций, переключение на альтернативные источники энергии, внедрение энергосберегающих технологий - лишь некоторые примеры применения метода реальных опционов в электроэнергетике, приведенные в данной статье. Помимо 
этого, реальные опционы могут также использоваться для определения структуры капитала компании (Lin, 2009), выбора времени IPO, проведения сделки M\&A и в других случаях. Исследование возможности применения реальных опционов в российских компаниях электроэнергетики остается темой предполагаемых исследований.

\section{Список литературы}

1. Дранев Ю.Я. О риск-нейтральном подходе ценообразования реальных опционов. // Корпоративные финансы. 2010. № 1 (13). С. 62-73.

2. Пирогов Н.К., Зубцов Н.Н. Взаимодействие реальных опционов на примере девелоперских проектов в России// Корпоративные финансы. 2008. № 6.

3. Benth, K. (2007), A Non-Gaussian Ornstein-Uhlenbeck Process for Electricity Spot Price Modeling and Derivatives Pricing, Applied Mathematical Finance, 14(2) (2007) 153-169.

4. Deng, S. (2003), Valuation of Electricity Generation Assets with Operational Characteristics, Probability in the Engineering and Informational Sciences (PEIS), 2003, April.

5. Driouchi, B. (2010), Real options in multinational decision-making: Managerial awareness and risk Implications, Journal of World Business, (2010).

6. Fuss, S., Szolgayova, J., Khabarov, N., Obersteiner, M. (2010), Renewables and climate change mitigation: Irreversible energy investment under uncertainty and portfolio effects, Energy Policy, In Press.

7. Gitelman, G. (2002), Use of real options in asset valuation, The Electricity Journal, 15(11) (2002) 58-71.

8. Hickey, E.A., Carlson, J.L., Loomis, D. (2010), Issues in the determination of the optimal portfolio of electricity supply options, Energy Policy, 38 (2010) 2198-2207.

9. Kulatilaka, N. (1993), The Value of Flexibility: The Case of a Dual-Fuel Industrial Steam Boiler, International Journal of Financial Management, Autumn (1993) 271-280.

10. Lin, T. (2009), The determinant of production entry and exit model on financing behavior, European Journal of Operational Research, 196 (2009) 258-265.

11. Lin, T., Huang, S.L. (2009), An entry and exit model on the energy - saving investment strategy with real options, Energy Policy, 3 (2009) 32-39.

12. Menegaki (2008), Valuation for renewable energy: A comparative review, Renewable and Sustainable Energy Reviews, 12 (2008) 2422-2437.

13. Naito, Takashima, Kimura, Madarame (2010), Evaluating replacement project of nuclear power plants under uncertainty, Energy Policy 38 (2010) 1321-1329.

14. Pindyck, R.S. (1988), Irreversible Investment, Capacity Choice, and the Value of the Firm, American Economic Review, 79 (1988) 969-985.

15. Szolgayova, J., Fuss, S., Obersteiner, M. (2008), Assessing the effects of CO2 price caps on electricity investments - A real options analysis, Energy Policy, 36 (2008) 3974-3981.

16. Schmit (2009), Ethanol plant investment using net present value and real options analyses, Biomass and Bioenergy, 33 (2009) 1442-1451.

17. Sanyal, P., Bulan, L. (2008) Regulatory Risk, Market Risk and Capital Structure: Evidence from U.S. Electric Utilities, Working paper.

18. Tseng, C., Barz, G. (2002), Short-term Generation Asset Valuation: A Real Options Approach, Operations Research, 50(2) (2002) 297-310.

19. Trigeorgis, L. (1996), Real Options: Managerial Flexibility and Strategy in Resource Allocation - Cambridge, MA.: MIT Press.

20. Verbruggen, A. (2009), Renewable energy costs, potentials, barriers: Conceptual issues, Energy Policy, 37 (2009) 5732-5743. 